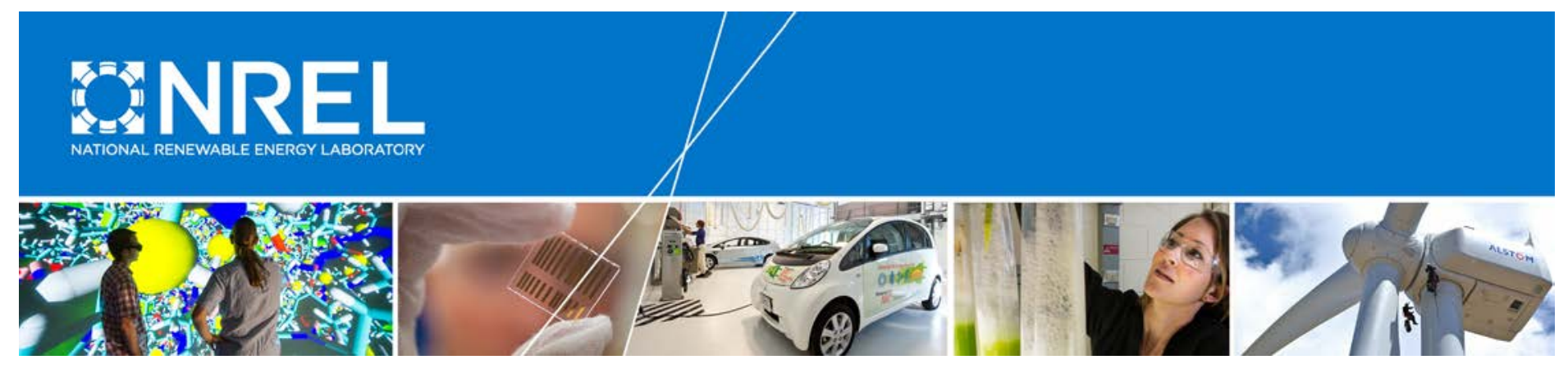

\title{
Local Voltage Control in Distribution Networks: A Game-Theoretic Perspective
}

\section{Preprint}

\author{
Xinyang Zhou, Jie Tian, and Lijun Chen \\ University of Colorado \\ Emiliano Dall'Anese \\ National Renewable Energy Laboratory
}

Presented at the 2016 North American Power Symposium (NAPS) Denver, Colorado

September 18-20, 2016

(C) 2016 IEEE. Personal use of this material is permitted. Permission from IEEE must be obtained for all other uses, in any current or future media, including reprinting/republishing this material for advertising or promotional purposes, creating new collective works, for resale or redistribution to servers or lists, or reuse of any copyrighted component of this work in other works.

NREL is a national laboratory of the U.S. Department of Energy Office of Energy Efficiency \& Renewable Energy Operated by the Alliance for Sustainable Energy, LLC

This report is available at no cost from the National Renewable Energy Laboratory (NREL) at www.nrel.gov/publications.

\section{Conference Paper}

NREL/CP-5D00-66847

September 2016 


\section{NOTICE}

The submitted manuscript has been offered by an employee of the Alliance for Sustainable Energy, LLC (Alliance), a contractor of the US Government under Contract No. DE-AC36-08GO28308. Accordingly, the US Government and Alliance retain a nonexclusive royalty-free license to publish or reproduce the published form of this contribution, or allow others to do so, for US Government purposes.

This report was prepared as an account of work sponsored by an agency of the United States government. Neither the United States government nor any agency thereof, nor any of their employees, makes any warranty, express or implied, or assumes any legal liability or responsibility for the accuracy, completeness, or usefulness of any information, apparatus, product, or process disclosed, or represents that its use would not infringe privately owned rights. Reference herein to any specific commercial product, process, or service by trade name, trademark, manufacturer, or otherwise does not necessarily constitute or imply its endorsement, recommendation, or favoring by the United States government or any agency thereof. The views and opinions of authors expressed herein do not necessarily state or reflect those of the United States government or any agency thereof.

This report is available at no cost from the National Renewable Energy Laboratory (NREL) at www.nrel.gov/publications.

Available electronically at SciTech Connect http:/www.osti.gov/scitech

Available for a processing fee to U.S. Department of Energy and its contractors, in paper, from:

U.S. Department of Energy

Office of Scientific and Technical Information

P.O. Box 62

Oak Ridge, TN 37831-0062

OSTI http://www.osti.gov

Phone: 865.576.8401

Fax: 865.576.5728

Email: reports@osti.gov

Available for sale to the public, in paper, from:

U.S. Department of Commerce

National Technical Information Service

5301 Shawnee Road

Alexandria, VA 22312

NTIS http://www.ntis.gov

Phone: 800.553 .6847 or 703.605 .6000

Fax: 703.605.6900

Email: orders@ntis.gov 


\title{
Local Voltage Control in Distribution Networks: A Game-Theoretic Perspective
}

\author{
Xinyang Zhou, Jie Tian, Lijun Chen, and Emiliano Dall'Anese
}

\begin{abstract}
Inverter-based voltage regulation is gaining importance to alleviate emerging reliability and power-quality concerns related to distribution systems with high penetration of photovoltaic (PV) systems. This paper seeks contribution in the domain of reactive power compensation by establishing stability of local Volt/VAr controllers. In lieu of the approximate linear surrogate used in the existing work, the paper establishes existence and uniqueness of an equilibrium point using nonlinear $\mathrm{AC}$ power flow model. Key to this end is to consider a nonlinear dynamical system with non-incremental local Volt/VAr control, cast the Volt/VAr dynamics as a game, and leverage the fixed-point theorem as well as pertinent contraction mapping argument. Numerical examples are provided to complement the analytical results.
\end{abstract}

Index Terms-Distribution systems, photovoltaic systems, voltage regulation, Volt/VAr control, game theory, stability.

\begin{tabular}{ll} 
& \multicolumn{1}{c}{ I. NOTATION } \\
$\mathcal{N}$ & set of buses excluding bus $0, \mathcal{N}:=\{1, \ldots, n\}$ \\
$\mathcal{L}$ & set of power lines \\
$\mathcal{L}_{i}$ & set of the lines from bus 0 to bus $\mathrm{i}$ \\
$\tilde{\mathcal{L}}_{i}$ & set of the lines descending from bus $\mathrm{i}$ \\
$p_{i}^{c}, q_{i}^{c}$ & real, reactive power consumption at bus $i$ \\
$p_{i}^{g}, q_{i}^{g}$ & real, reactive power generation at bus $i$ \\
$P_{i j}, Q_{i j}$ & real and reactive power flow from $i$ to $j$ \\
$r_{i j}, x_{i j}$ & resistance and reactance of line $(i, j)$ \\
$v_{i}$ & squared magnitude of complex voltage at bus $i$ \\
$\ell_{i j}$ & squared magnitude of complex current of \\
{$[x]^{+}$} & line $(i, j)$ \\
{$[x]_{\Omega_{i}}$} & positive part, $x^{+}=$maxection of $x$ onto the set $\{0, x\}$
\end{tabular}

A quantity without subscript is usually a vector with appropriate components defined earlier, e.g., $v:=\left(v_{i}, i \in \mathcal{N}\right), q^{g}:=$ $\left(q_{i}^{g}, i \in \mathcal{N}\right)$.

\section{INTRODUCTION}

Capacitor banks and load tap changers are traditionally utilized to control voltage levels across a distribution system [1][3]. Given the predictable and slow changes in demand in traditional operational conditions, switching operations were required only a few times per day. However, with the increasing photovoltaic (PV) capacity in both residential and commercial setting, the increased likelihood of overvoltage conditions due to reverse power flows and rapid fluctuations in generation call for new voltage control paradigms. Even though the current IEEE Standard 1547 requires distributed

X. Zhou, J. Tian and L. Chen are with College of Engineering and Applied Science, University of Colorado, Boulder, CO 80309, USA (emails: \{xinyang.zhou, lijun.chen\}@colorado.edu, tianliudou@gmail.com).

E. Dall'Anese is with National Renewable Energy Laboratory, Golden, CO 80401, USA (email: emiliano.dallanese@nrel.gov). generation to operate at unity power factor, PV-inverters can readily adjust real and reactive power outputs to stabilize voltages and cope with fast time-varying conditions. One way to regulate voltages within the given limits consists of implementing at the PV inverters local Volt/VAr control mechanisms to adjust the output reactive power based on current voltage levels at the point of connection [4]-[7]. In lieu of local control rules, optimization based strategies involve a joint control of both real and reactive power injection based on given optimization objectives; see, e.g., [16], [17].

Consistent with the IEEE 1547.8 Standard [12], [13], inverter-based local Volt/VAr control schemes were investigated in, e.g., [8]-[11]. Particularly, the linearized dynamical systems with reactive power control outlined in [8]-[10] have being shown to have a unique equilibrium point that coincides with the unique optimal solution of a well-defined convex optimization problem. With the objective of driving voltage values at equilibrium to within a given range, [11] proposed an incremental Volt/VAr strategy that does not modify the reactive power when the node voltage is within the prescribed range. The resultant dynamical system has been shown to solve an optimization problem that minimizes a cost of voltage deviation.

However, these control strategies are grounded on a linearized AC power flow model for mathematical tractability. With a linearized model, voltage values can be approximated as a linear function of reactive power injections [8]. In contrast, this paper seeks an analytical characterization of Volt/VAr control using exact nonlinear AC power flow models. To this end, the paper utilizes a reverse-engineering approach to cast the nonlinear dynamical system with non-incremental Volt/VAr control as a game, where each node acts as a "selfish player" who uses its local control function as a bestresponse strategy to minimize its own cost function. Using this approach, we show that the equilibrium of the Volt/VAr control dynamics is equivalent to the equilibrium of the resulting game. We further prove the existence and uniqueness of the equilibrium by leveraging the fixed-point theorem as well as contraction mapping argument.

The analysis is also extended to account for incremental Volt/VAr controls. Particularly, we show that a few incremental controls can be reverse-engineered as a distributed strategies for solving the well-defined voltage control game or a distributed algorithm for solving an optimization problem. Existence and convergence of the equilibria can again be established through the contraction mapping argument.

The rest of this paper is organized as follows. Section III outlines the nonlinear power flow model and the non-incremental local Volt/VAr control. Section IV reverseengineers the nonlinear control dynamical system as a voltage 
control game and proves the existence and uniqueness of its equilibrium. Section $\mathrm{V}$ extends the result to a few incremental local Volt/VAr controls in nonlinear system. Section VI provides numerical examples to complement the analytical results, and Section VII concludes the paper.

\section{System MODEL}

\section{A. Power flow model}

Consider a radial distribution network, modeled as a directed and connected tree graph $(\mathcal{N} \cup\{0\}, \mathcal{L})$, with $|\mathcal{N}|=$ $|\mathcal{L}|=n$. To represent the AC power flow, we use the following branch flow model [1], [14]:

$$
\begin{aligned}
P_{i j} & =p_{j}^{c}-p_{j}^{g}+\sum_{k:(j, k) \in \mathcal{L}} P_{j k}+r_{i j} \ell_{i j}, \\
Q_{i j} & =q_{j}^{c}-q_{j}^{g}+\sum_{k:(j, k) \in \mathcal{L}} Q_{j k}+x_{i j} \ell_{i j}, \\
v_{j} & =v_{i}-2\left(r_{i j} P_{i j}+x_{i j} Q_{i j}\right)+\left(r_{i j}^{2}+x_{i j}^{2}\right) \ell_{i j}, \\
\ell_{i j} v_{i} & =P_{i j}^{2}+Q_{i j}^{2} .
\end{aligned}
$$

Notice that $v_{0}, p^{c}, p^{g}, q^{c}$ are given constants, and that reactive powers $q^{g}:=\left(q_{1}^{g}, \ldots, q_{n}^{g}\right)$ are control variables. For notational simplicity, in the rest of the paper we will ignore the superscript in $q^{g}$ and write $q$ instead. Further, let $\Omega_{i}:=$ $\left\{q_{i} \mid q_{i}^{\min } \leq q_{i} \leq q_{i}^{\max }\right\}$ denote the set of available reactive power injection at bus $i$, and define $\Omega:=\prod_{i \in \mathcal{N}} \Omega_{i}$.

The power flow equations (1) can be represented in the following compact form:

$$
F(P, Q, \ell, v, q)=0,
$$

where $F$ is twice continuously differentiable with respect to $q$ and $y:=(P, Q, \ell, v)$. For prevailing ambient conditions, given the reactive powers $q, y$ is uniquely determined for distribution networks setups where $v_{0} \approx 1$ and $r_{i j}, x_{i j}$ are sufficiently small [3]. It has also been shown in [3] (Proposition 4-1) that, under the setup of $v_{0} \approx 1$ and small $r_{i j}, x_{i j}$, the Jacobian matrix $\partial_{y} F(y, q)$ is nonsingular for a topology of one main feeder with its direct laterals. This result can be straightforwardly extended to general radial networks where laterals may have laterals and so on, because the corresponding expanded Jacobian matrix keeps the same crucial properties for both its diagonal and off-diagonal blocks. We therefore focus on the setup of $v_{0} \approx 1$ and small $r_{i j}, x_{i j}$ in this paper. Then, by the implicit function theorem, it follows that equation (2) (i.e., equations (1)) defines implicitly a twice continuously differentiable function $y=y(q)$. Since $\Omega$ is compact, $\partial_{q} y(q)$ is bounded uniformly on $\Omega$, i.e., the first-order derivatives of $P, Q, \ell, v$ with respect to $q$ are all bounded on $\Omega$.

Consider representing the voltage magnitude as a function of the reactive powers $q$ in the following compact form:

$$
v=v(q),
$$

and notice that $\frac{\partial v_{i}}{\partial q_{j}}$ is bounded $\forall i, j \in \mathcal{N}$. Define the reactance matrix $X=\left[X_{i j}\right]_{n \times n}$ with entries

$$
X_{i j}:=\sum_{(h, k) \in \mathcal{L}_{i} \cap \mathcal{L}_{j}} 2 \cdot x_{h k}>0 .
$$

It can be readily checked that the matrix $X$ is symmetric. In the following, we will particularly relate the derivative of $v$ with respect to $q$ to the matrix $X$, which will be useful for the analysis in Section IV-B.

Lemma 1 In a radial distribution system, for $\forall i, j \in \mathcal{N}$, we have

$$
\left|\frac{\partial v_{i}}{\partial q_{j}}\right| \leq(1+\eta) X_{i j}
$$

for some $\eta>0$.

Proof: In [8], we have shown that for the linearized and lossless power flow models (i.e., terms involving $\ell_{l k}, \forall(l, k) \in$ $\mathcal{L}$, are all set to zero), the approximated voltage value, denoted $\hat{v}$, satisfies:

$$
\hat{v}=X q+\tilde{v}
$$

where $\tilde{v}$ is a constant determined by the system setup.

Considering the nonlinear model, we add up (1c) from any node $i \in \mathcal{N}$ all the way back to node 0 to obtain:

$$
\begin{aligned}
v_{i}= & v_{0}+\sum_{(l, k) \in \mathcal{L}_{i}}\left(2\left(r_{l k} P_{l k}+x_{l k} Q_{l k}\right)-\left(r_{l k}^{2}+x_{l k}^{2}\right) \ell_{l k}\right) \\
= & \hat{v}_{i}+\underset{(l, k) \in \mathcal{L}_{i}}{ }\left(r_{l k} \sum_{(r, s) \in \tilde{\mathcal{L}}_{k}} r_{r s} \ell_{r s}\right)+2 \sum_{(l, k) \in \mathcal{L}_{i}}\left(x_{l k} \sum_{(r, s) \in \tilde{\mathcal{L}}_{k}} x_{r s} \ell_{r s}\right) \\
& -\sum_{(l, k) \in \mathcal{L}_{i}}\left(r_{l k}^{2}+x_{l k}^{2}\right) \ell_{l k} .
\end{aligned}
$$

Let $G_{i}(\ell)$ denote the three summation terms on the right-hand side of (6), and substitute $\hat{v}$ with (5). Then equation (6) takes a simpler form of

$$
v_{i}=\sum_{m \in \mathcal{N}} X_{i m} q_{m}+\tilde{v}_{i}+G_{i}(\ell) .
$$

Notice that $G_{i}(\ell)$ is a function of single variable $\ell$ with an order of one. Take derivative of (7) on both sides with respect to $q_{j}$ from any bus $j \in \mathcal{N}$, and we have

$$
\frac{\partial v_{i}}{\partial q_{j}}=X_{i j}+\frac{\partial G_{i}(\ell)}{\partial q_{j}}
$$

and thus

$$
\left|\frac{\partial v_{i}}{\partial q_{j}}\right| \leq X_{i j}+\left|\frac{\partial G_{i}(\ell)}{\partial q_{j}}\right|
$$

Since $\left|\frac{\partial \ell_{l k}}{\partial q_{j}}\right|$ is bounded and $X_{i j}$ is nonzero, ${ }^{1}$ there exists an $\eta_{i j}>0$ such that

$$
\left|\frac{\partial G_{i}(\ell)}{\partial q_{j}}\right| \leq \eta_{i j} \cdot X_{i j}
$$

Take $\eta=\max _{i, j \in \mathcal{N}} \eta_{i j}$, and the inequality (4) follows.

Remarks: An accurate characterization of $\eta$ is challenging. In the numerical experiments, we have found that $\eta$ is usually a small number. For example, $\eta \leq 0.2$ with the setup of the 42-bus distribution network used in Section VI. This is due to the fact that $\left|\frac{\partial \ell_{l k}}{\partial q_{j}}\right|$ is bounded, and that $r_{l k}, x_{l k}$ are small.

\footnotetext{
${ }^{1}$ Here we have assumed that, without loss of generality, the bus 0 has only one child node. Notice that, when the bus 0 has multiple child nodes, as the squared voltage magnitude $v_{0}$ is a constant, the branches of different child nodes are independent
} 


\section{B. Local Volt/VAr control}

The goal of Volt/VAr control on a distribution network is to adjust the output reactive powers $q:=\left(q_{1}, \ldots, q_{n}\right)$ in order to maintain the node voltages $v:=\left(v_{1}, \ldots, v_{n}\right)$ within a given range around their nominal values $v_{i}^{\text {nom }}, i \in \mathcal{N}$ (e.g., ANSI C.84.1 limits). Volt/VAr can be modeled as a feedback control mechanism with state $(v(t), q(t))$, where the current state $(v(t), q(t))$ is mapped to a new reactive power injections $q(t+1)$. Usually $q(t+1)$ is determined either completely or partly by a certain Volt/VAr control function defined as follows.

Definition 1 A Volt/VAr control function $f: \mathbb{R}^{n} \rightarrow \mathbb{R}^{n}$ is a collection of $f_{i}: \mathbb{R} \rightarrow \mathbb{R}$ functions, each mapping the current local voltage $v_{i}$ to a local control variable $o_{i}$ in reactive power at node $i$ :

$$
o_{i}=f_{i}\left(v_{i}\right), \quad \forall i \in \mathcal{N} .
$$

The control functions $f_{i}$ are usually decreasing but not always strictly decreasing because of a deadband. Assume that for each node $i \in \mathcal{N}$ a symmetric deadband around the nominal voltage $\left(v_{i}^{\text {nom }}-\delta_{i} / 2, v_{i}^{\text {nom }}+\delta_{i} / 2\right)$ with $\delta_{i} \geq 0$ is utilized. Further, the following two assumptions are made (see e.g., [8]):

A1: The Volt/VAr control functions $f_{i}$ are non-increasing over $\mathbb{R}$ and strictly decreasing and differentiable in $\left(-\infty, v_{i}^{\text {nom }}-\delta_{i} / 2\right)$ and in $\left(v_{i}^{\text {nom }}+\delta_{i} / 2,+\infty\right)$.

A2: The derivative of the control function $f_{i}$ is bounded, i.e., there exists a finite $\alpha_{i}$ such that $\left|f_{i}^{\prime}\left(v_{i}\right)\right| \leq \alpha_{i}$ for all $v_{i}$ in the appropriate domain.

See Fig. 1 for an illustrative example of a piecewise linear droop control function

$$
f_{i}\left(v_{i}\right):=-\alpha_{i}\left[v_{i}-v_{i}^{\mathrm{nom}}-\frac{\delta_{i}}{2}\right]^{+}+\alpha_{i}\left[-v_{i}+v_{i}^{\mathrm{nom}}-\frac{\delta_{i}}{2}\right]^{+} .
$$

Equation (3) together with the control function (9) yields the following dynamical system for Volt/VAr control:

$$
\begin{aligned}
v(t) & =v(q(t)), \\
q(t+1) & =[f(v(t))]_{\Omega},
\end{aligned}
$$

with locally measured $v(t)$ as the only control input, and $q(t)$ the only control variables.

Definition 2 A point $\left(q^{*}, v^{*}\right)$ is called an equilibrium, if it is a fixed point of the dynamical system (11), i.e.,

$$
\begin{aligned}
v^{*} & =v\left(q^{*}\right), \\
q^{*} & =\left[f\left(v^{*}\right)\right]_{\Omega} .
\end{aligned}
$$

\section{A VOLTAGE CONTROL GAME}

We have shown in [8] that, given voltage $v_{i}(t)$, the reactive power $q_{i}(t+1)$ in (11b) is the unique solution of the following optimization problem:

$$
q_{i}(t+1)=\underset{q_{i} \in \Omega_{i}}{\arg \min } u_{i}\left(q_{i} ; v_{i}(t)\right),
$$

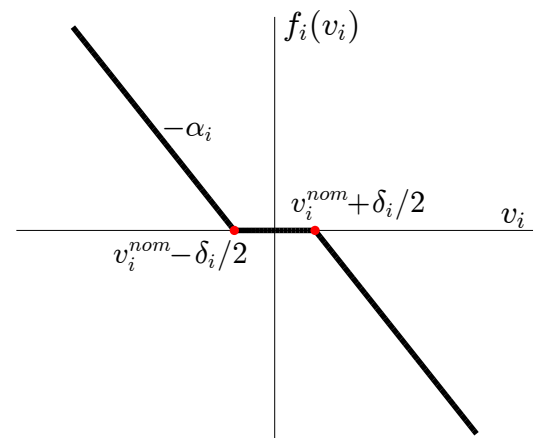

Fig. 1: Piecewise linear Volt/VAr control curve $f_{i}$.

where

$$
u_{i}\left(q_{i} ; v_{i}\right):=C_{i}\left(q_{i}\right)+q_{i} v_{i}
$$

with $C_{i}\left(q_{i}\right):=-\int_{0}^{q_{i}} f_{i}^{-1}(q) d q$ is a convex function since $f_{i}^{-1}$ is non-increasing under A1. This result motivates us to cast the dynamics (11) as a game as shown next.

\section{A. Voltage control game}

We view each node $i \in \mathcal{N}$ as a player with strategy space $\Omega_{i}$ and a cost function $u_{i}\left(q_{i} ; v_{i}(q)\right)$ defined by (14). Recalling that $\Omega:=\prod_{i \in \mathcal{N}} \Omega_{i}$, the voltage control game is defined next.

Definition 3 A non-cooperative voltage control game is defined as a triple $\mathcal{G}_{v c}:=\left\{\mathcal{N}, \Omega,\left(u_{i}\left(q_{i} ; v_{i}(q)\right)\right)_{i \in \mathcal{N}}\right\}$, where the strategic interaction among players is through the voltage $v_{i}(q), i \in \mathcal{N}$.

In consistence with the introduction of the cost function $u_{i}$, we need to extend the concept of usual Nash equilibrium.

Definition 4 An equilibrium of the voltage control game $\mathcal{G}_{v c}$ is a tuple $\left(q^{*} ; v^{*}\right)$ such that $\forall i \in \mathcal{N}, q_{i}^{*}$ is the best response to $v_{i}\left(q^{*}\right)$, i.e., for $\forall i \in \mathcal{N}$,

$$
\begin{aligned}
u_{i}\left(q_{i}^{*} ; v_{i}^{*}\right) & \leq u_{i}\left(q_{i} ; v_{i}^{*}\right), \forall q_{i} \in \Omega_{i}, \\
v_{i}^{*} & =v_{i}\left(q^{*}\right) .
\end{aligned}
$$

In the above definition of equilibrium, the buses respond directly to given voltages. Similar to the price-taking behavior of the agents in a competitive market, we call such a behavior here signal-taking, i.e., when a bus makes decisions, it takes the voltage $v_{i}$ as given but does not take into consideration the impact of its own decision upon the voltages. ${ }^{2}$

Recall that $v_{i}(q)$ is the implicit function from the implicit equation (2) (i.e., the power flow equations (1)), the following result is immediate.

Theorem 1 The dynamical system (11) can be viewed as the best response algorithm for the voltage control game $\mathcal{G}_{v c}$. Moreover, a point $\left(q^{*}, v^{*}\right)$ is an equilibrium of (11) if and only if $\left(q^{*}, v^{*}\right)$ is an equilibrium of $\mathcal{G}_{v c}$.

We further show the existence of the equilibrium of $\mathcal{G}_{v c}$.

\footnotetext{
${ }^{2}$ When a bus takes into consideration the impact of its own decision upon the voltages, we say that this behavior is signal-anticipating [15]. With signalanticipating buses, we can define the usual Nash equilibrium for the voltage control game, which we will investigate in another paper.
} 
Theorem 2 Given the continuous Volt/VAr control functions $f(\cdot)$, there exists an equilibrium for the voltage control game $\mathcal{G}_{v c}$.

Proof: Recalling from Section III that $v(q)$ is continuously differentiable, we know that the best response algorithm of the game $\mathcal{G}_{v c}$, i.e., the dynamical system (11), is a continuous differentiable mapping from $\Omega$ to itself. Since $\Omega$ is compact, by Brouwer's fixed-point theorem there exists an equilibrium for the game $\mathcal{G}_{v c}$.

\section{B. Convergence of dynamics and uniqueness of equilibrium}

In this subsection, we establish a sufficient condition for convergence of the dynamical system (11) by leveraging the pertinent contraction mapping. Existence and uniqueness of its equilibrium will follow.

To this end, consider rewriting the control dynamics using the following mapping $g: \Omega \rightarrow \Omega$ as

$$
q(t+1)=[f(v(q(t)))]_{\Omega}:=g(q(t)) .
$$

\section{Lemma 2 If condition}

$$
\alpha_{i}(1+\eta) \sum_{j} X_{i j}<1, \forall i \in \mathcal{N}
$$

holds, then the mapping $g$ is a contraction mapping.

Proof: Define $\tilde{X}=\left[\tilde{X}_{i j}\right]_{n \times n}$ with its entries $\tilde{X}_{i j}=$ $\frac{\partial v_{i}}{\partial q_{j}}, \forall i, j \in \mathcal{N}$. With induced matrix norm $\|\cdot\|_{\infty}$ as the maximum row sum, we have

$$
\begin{aligned}
\left\|\nabla_{q} g\right\|_{\infty} & \leq \max _{i}\left(\left|f_{i}^{\prime}\left(v_{i}\right)\right| \sum_{j}\left|\tilde{X}_{i j}\right|\right) \\
& \leq \max _{i}\left(\alpha_{i} \sum_{j}\left|\tilde{X}_{i j}\right|\right) \\
& \leq \max _{i}\left(\alpha_{i}(1+\eta) \sum_{j} X_{i j}\right) \\
& <1,
\end{aligned}
$$

where the four inequalities respectively come from 1) the possibility of $q$ 's being projected onto the boundary of $\Omega$, making the corresponding derivative of $g$ equal to zero (as well as a very small chance for $\tilde{X}_{i j}$ to be negative), 2) assumption A2, 3) Lemma 1, and 4) condition (16). Hence, given $\forall q_{x}, q_{y} \in \Omega$, we have

$\left\|g\left(q_{x}\right)-g\left(q_{y}\right)\right\|_{\infty} \leq\left\|\frac{\partial g}{\partial q}\right\|_{\infty} \cdot\left\|q_{x}-q_{y}\right\|_{\infty}<\left\|q_{x}-q_{y}\right\|_{\infty}$,

i.e., $g$ is a contraction mapping.

Then, using the contraction mapping theorem [19], the following result can be demonstrated.

Theorem 3 Under the condition (16), the dynamics (11) converges to the unique equilibrium point.

Remarks: Notice that when $\eta=0$, i.e., when we ignore line loss, (16) coincides with the convergence condition for the same control strategy with linearized model in [8]. While it is challenging to characterize $\eta$, as will be shown by numerical examples in Section VI-B, by setting $\eta=0$, we usually still have a practical sufficient convergence condition for non-linear model, because (16) is a conservative condition, and that $\eta$ itself is normally small.

\section{EXTENSIONS TO INCREMENTAL CONTROLS}

\section{A. Incremental local volt/var controls}

In addition to the non-incremental local Volt/VAr control (11b), we have in previous works proposed three incremental local Volt/Var control algorithms under linearized model [9][11], and proved their stability. We list these control algorithms as follows with fixed stepsize $\gamma_{g}, \gamma_{p}, \gamma_{v}$ respectively:

$$
\begin{aligned}
q_{i}(t+1) & =\left[q_{i}(t)-\gamma_{g}\left(v_{i}(q(t))-f_{i}^{-1}\left(q_{i}\right)\right)\right]_{\Omega_{i}}, \\
q_{i}(t+1) & =\left[q_{i}(t)-\gamma_{p}\left(q_{i}(t)-f_{i}\left(v_{i}(q(t))\right)\right)\right]_{\Omega_{i}}, \\
q_{i}(t+1) & =\left[q_{i}(t)+\gamma_{v} f_{i}\left(v_{i}(q(t))\right)\right]_{\Omega_{i}} .
\end{aligned}
$$

By replacing (11b) from dynamics (11) with (17), (18), and (19) respectively, we can obtain three non-linear dynamical systems. We will show next that these incremental algorithms within nonlinear power flow model preserve their stability.

\section{B. Reverse-engineering, equilibrium, and convergence}

Since (11b), (17), and (18) can be seen respectively as best-response algorithm, (sub)gradient algorithm, and pseodogradient algorithm for solving the same minimization problem (13), all three dynamical systems (11a)-(11b), (11a)(17) and (11a)(18) can be reverse-engineered as the same voltage control game $\mathcal{G}_{v c}$. We can again through contraction mapping give sufficient convergence conditions, under which the dynamical systems (11a)(17) and (11a)(18) converge to the unique equilibrium.

As for dynamics (11a)(19), we first define the following voltage control optimization problem:

$$
\min _{q} \sum_{i \in \mathcal{N}}-\int_{0}^{v_{i}(q)} f_{i}(v) d v .
$$

Although this is a non-convex problem, it is easy to check that its KKT conditions coincide with equilibrium conditions of (11a)(19). Similarly through showing contraction mapping, convergence condition can be given to ensure that this dynamical system converges to acceptable voltage ranges determined by deadband of control functions. It also indicates the possibility of infinitely many equilibria for (11a)(19).

These results are consistent with both analytical (with linearized model) and numerical (with nonlinear model) results in our previous works [9]-[11] with linear model.

\section{NUMERICAL EXAMPLES}

We now provide numerical examples to complement the theoretical analysis in previous sections. 


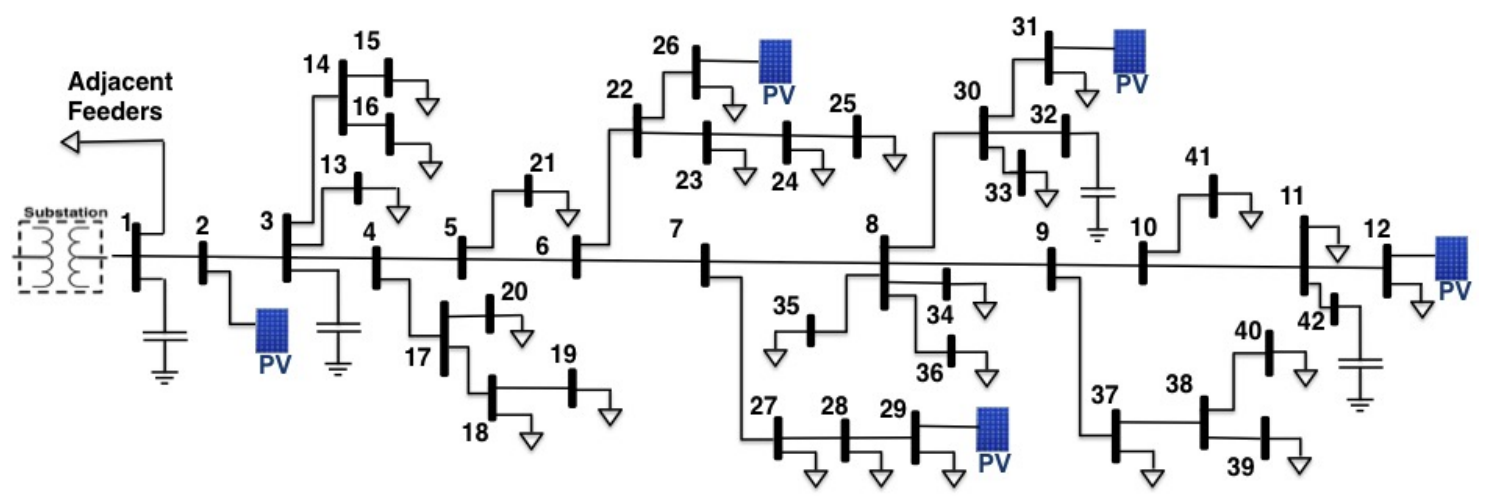

Fig. 2: Circuit diagram for a SCE distribution system.

TABLE I: Network of Fig. 2: Line impedances, peak spot load KVA, Capacitors and PV generation's nameplate ratings.

\begin{tabular}{|c|c|c|c|c|c|c|c|c|c|c|c|c|c|c|c|c|c|}
\hline \multicolumn{18}{|c|}{ Network Data } \\
\hline \multicolumn{4}{|c|}{ Line Data } & \multicolumn{4}{|c|}{ Line Data } & \multicolumn{4}{|c|}{ Line Data } & \multicolumn{2}{|c|}{ Load Data } & \multicolumn{2}{|c|}{ Load Data } & \multicolumn{2}{|c|}{ PV Generators } \\
\hline $\begin{array}{l}\text { From } \\
\text { Bus. }\end{array}$ & $\begin{array}{l}\text { To } \\
\text { Bus. }\end{array}$ & $\begin{array}{c}\mathrm{R} \\
(\Omega)\end{array}$ & $\begin{array}{c}\mathrm{X} \\
(\Omega)\end{array}$ & $\begin{array}{l}\text { From } \\
\text { Bus. }\end{array}$ & $\begin{array}{r}\text { To } \\
\text { Bus }\end{array}$ & $\begin{array}{c}\mathrm{R} \\
(\Omega)\end{array}$ & $\begin{array}{c}\mathrm{X} \\
(\Omega)\end{array}$ & $\begin{array}{c}\text { From } \\
\text { Bus. }\end{array}$ & $\begin{array}{c}\text { To } \\
\text { Bus. }\end{array}$ & $\begin{array}{c}\mathrm{R} \\
(\Omega)\end{array}$ & $\begin{array}{c}\mathrm{X} \\
(\Omega)\end{array}$ & $\begin{array}{l}\text { Bus } \\
\text { No. }\end{array}$ & $\begin{array}{l}\text { Peak } \\
\text { MVA }\end{array}$ & $\begin{array}{l}\text { Bus } \\
\text { No. }\end{array}$ & $\begin{array}{l}\text { Peak } \\
\text { MVA }\end{array}$ & $\begin{array}{l}\text { Bus } \\
\text { No. }\end{array}$ & $\begin{array}{c}\text { Capacity } \\
\text { MW }\end{array}$ \\
\hline 1 & 2 & 0.259 & 0.808 & 8 & 34 & 0.244 & 0.046 & 18 & 19 & 0.198 & 0.046 & 11 & 0.67 & 28 & 0.27 & & \\
\hline 2 & 3 & 0.031 & 0.092 & 8 & 36 & 0.107 & 0.031 & 22 & 26 & 0.046 & 0.015 & 12 & 0.45 & 29 & 0.2 & 2 & 1.05 \\
\hline 3 & 4 & 0.046 & 0.092 & 8 & 30 & 0.076 & 0.015 & 22 & 23 & 0.107 & 0.031 & 13 & 0.89 & 31 & 0.27 & 26 & 2.1 \\
\hline 3 & 13 & 0.092 & 0.031 & 8 & 9 & 0.031 & 0.031 & 23 & 24 & 0.107 & 0.031 & 15 & 0.07 & 33 & 0.45 & 29 & 1.89 \\
\hline 3 & 14 & 0.214 & 0.046 & 9 & 10 & 0.015 & 0.015 & 24 & 25 & 0.061 & 0.015 & 16 & 0.67 & 34 & 1.34 & 31 & 2.625 \\
\hline 4 & 17 & 0.336 & 0.061 & 9 & 37 & 0.153 & 0.046 & 27 & 28 & 0.046 & 0.015 & 18 & 0.45 & 35 & 0.13 & 12 & 3.15 \\
\hline 4 & 5 & 0.107 & 0.183 & 10 & 11 & 0.107 & 0.076 & 28 & 29 & 0.031 & 0 & 19 & 1.23 & 36 & 0.67 & & \\
\hline 5 & 21 & 0.061 & 0.015 & 10 & 41 & 0.229 & 0.122 & 30 & 31 & 0.076 & 0.015 & 20 & 0.45 & 37 & 0.13 & & \\
\hline 5 & 6 & 0.015 & 0.031 & 11 & 42 & 0.031 & 0.015 & 30 & 32 & 0.076 & 0.046 & 21 & 0.2 & 39 & 0.45 & & \\
\hline 6 & 22 & 0.168 & 0.061 & 11 & 12 & 0.076 & 0.046 & 38 & 39 & 0.107 & 0.015 & 23 & 0.13 & 40 & 0.2 & & \\
\hline 6 & 7 & 0.031 & 0.046 & 14 & 16 & 0.046 & 0.015 & 38 & 40 & 0.061 & 0.015 & 24 & 0.13 & 41 & 0.45 & & \\
\hline 7 & 27 & 0.076 & 0.015 & 14 & 15 & 0.107 & 0.015 & 43 & 44 & 0.061 & 0.015 & 25 & 0.2 & & $V_{\text {base }}$ & 12.3 & \\
\hline 7 & 8 & 0.015 & 0.015 & 17 & 18 & 0.122 & 0.092 & 43 & 45 & 0.061 & 0.015 & 26 & 0.07 & & $S_{\text {base }}$ & 1000 & \\
\hline 8 & 35 & 0.046 & 0.015 & 17 & 20 & 0.214 & 0.046 & & & & & 27 & 0.13 & & $Z_{\text {base }}$ & 152. & \\
\hline
\end{tabular}
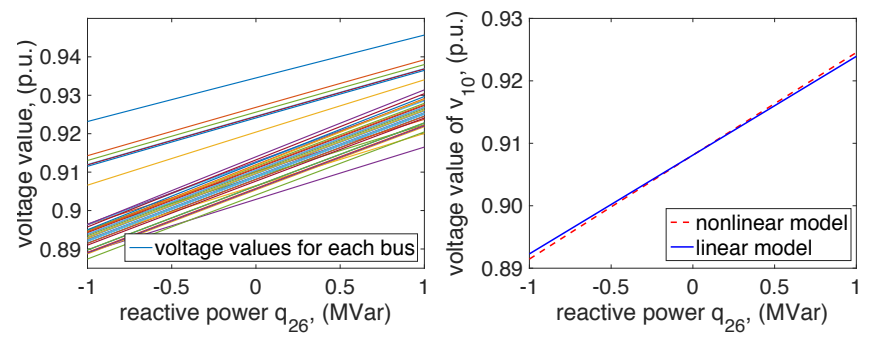

Fig. 3: (Left) Voltage values of all buses as reactive power injection from Bus 26 changes; (right) voltage values of Bus 10 in both linear and non-linear models as reactive power injection from Bus 26 changes.

\section{A. Simulation Setup}

The network topology (Fig. 2) and its parameters (TABLE I) are based on a distribution feeder of South California Edison. As shown in Fig. 2, Bus 1 is actually the reference " 0 " bus, and a total of five PVs are installed at Bus 2, 12, 26, 29, and 31 respectively. ${ }^{3}$ AC power flow model (1) is calculated by MatLab tool MATPOWER 5.1 [18].

We use the piecewise linear droop control functions (10) with their slopes $\alpha_{i}$ to be determined and analyzed. We assume

\footnotetext{
${ }^{3}$ Different from what is implied in the previous sections, in practice we may not have PVs at all buses. As a result, the convergence condition needs to be tailored accordingly.
}

that all the control functions have identical acceptable voltage range $\left[0.98^{p . u .}, 1.02^{p . u \cdot}\right]$, i.e., $\delta_{i}=0.04^{p . u .}, \forall i \in \mathcal{N}$.

\section{B. Effects of reactive power injections upon voltage values}

In this part, we examine how voltage values change with different reactive injections. We fix the reactive power injections of all inverters as 0 except that at Bus 26. We sweep the reactive power injections at Bus 26 from $-1 \mathrm{MW}$ to $1 \mathrm{MW}$ with granularity of $0.1 \mathrm{MW}$, and record the consequent voltage changes at all buses. Similar results are observed by engaging any other inverters.

According to (8), we do not preclude the possibility of negative $\frac{\partial v_{i}}{\partial q_{i}}$, but it rarely takes place since the second term in (8) is usually much smaller than $X_{i j}$, almost always resulting in positive $\frac{\partial v_{i}}{\partial q_{j}}, \forall i, j \in \mathcal{N}$, as we can see from the illustration in Fig. 3 (left).

We then arbitrarily pick Bus 10 to compare its voltage changes against reactive power injections in both nonlinear and linear models. As illustrated in Fig. 3 (right), the slopes exhibiting $\frac{\partial v_{10}}{\partial q_{26}}$ in two models are very close. We can find a small parameter $\eta$, such that the slope in nonlinear model is upper-bounded by that in linear model multiplied by $(1+\eta)$. In this case, $\eta$ can be set as 0.2 . Similar results are observed from voltage values of any other buses and reactive power injections from any other inverters, though the slope in nonlinear model 
(a) $\max \alpha_{\mathrm{i}}=11$

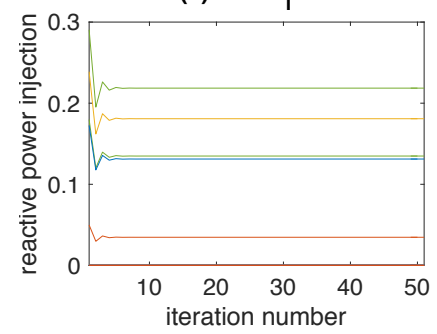

(c) $\max \alpha_{i}=32$
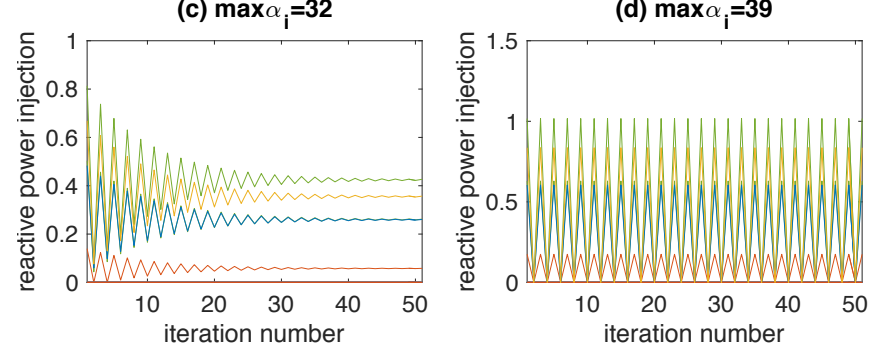

Fig. 4: As the values of $\alpha_{i}$ increase from (a) to (d), we have slower convergence speed and finally reach a non-convergent result, when the values of $\alpha_{i}$ get too large.

is not necessarily greater than that in linearized model due to the possibility of $\frac{\partial \ell_{l k}}{\partial q_{j}}$ being negative.

\section{Convergence}

In this part, we set different $\alpha_{i}$ values in the piecewise linear droop control functions to see how they affect the convergence of our Volt/VAr control dynamics (11). As illustrated in Fig. 4 , when we increase simultaneously the values of slopes for all five droop control functions from Fig. 4(a) to Fig. 4(d), we observe decreasing convergence speeds, until convergence is no longer available with too large $\alpha_{i}$, where oscillation occurs (Fig. 4(d)).

From simulation results, we also observe that, the maximum allowed slope value $\max \alpha_{i} \approx 33$, is much larger than the $\alpha<4.6$ upper bound ${ }^{i}$ calculated by convergence condition (16) with $\eta=0$. With larger $\eta$, we get even smaller sufficient upper bound. This is because the sufficient condition (16) is conservative estimation.

\section{CONCLUSION}

In order to analytically characterize the equilibrium and convergence of the local Volt/VAr control dynamics with nonlinear power flow model, we reverse-engineer the dynamical system with non-incremental control as a voltage control game. We then establish the existence, uniqueness, and convergence of the equilibrium by the fixed-point theorem and pertinent contraction mapping argument. We also extend the results to the incremental Volt/VAr controls. Numerical examples are provided to complement the analytical results.

\section{ACKNOWLEDGMENT}

We thank Guohui Ding, Zhiyuan Liu, and Wanshan Yang at University of Colorado, Boulder for helpful discussions.

\section{REFERENCES}

[1] M. E. Baran and F. F Wu. Optimal capacitor placement on radial distribution systems. IEEE Trans. Power Delivery, 4(1):725-734, 1989.

[2] M. E Baran and F. F Wu. Optimal sizing of capacitors placed on a radial distribution System. IEEE Trans. Power Delivery, 4(1):735-743, 1989.

[3] H-D. Chiang and M. E. Baran. On the existence and uniqueness of load flow solution for radial distribution power networks. IEEE Trans. Circuits and Systems, 37(3):410-416, March 1990.

[4] M. Farivar, C. R. Clarke, S. H. Low, and K. M. Chandy. Inverter var control for distribution systems with renewables. Proceedings of IEEE SmartGridComm Conference, October 2011.

[5] J. W. Smith, W. Sunderman, R. Dugan, and B. Seal. Smart inverter volt/var control functions for high penetration of PV on distribution systems. IEEE PES Power Systems Conference and Exposition (PSCE), 2011.

[6] K. Turitsyn, P. Sŭlc, S. Backhaus, and M. Chertkov. Options for control of reactive power by distributed photovoltaic generators. Proc. of the IEEE, 99(6):1063 -1073, June 2011.

[7] P. Jahangiri and D. C. Aliprantis. Distributed volt/vAr control by PV inverters. IEEE Trans. Power System, vol. 28, no. 3, pp. 3429-3439, Aug. 2013.

[8] M. Farivar, L. Chen, and S. Low. Equilibrium and dynamics of local voltage control in distribution systems. 52nd IEEE Annual Conference on Decision and Control (CDC), pp. 4329-4334, 2013.

[9] M. Farivar, X. Zhou, and L. Chen. Local voltage control in distribution system: An incremental control algorithm. Proc. IEEE Intl. Conf. on Smart Grid Commun, 2015.

[10] X. Zhou, M. Farivar, and L. Chen. Pseudo-gradient based local voltage control in distributed networks. 53nd Annual Allerton Conference on Communication, Control, and Computing, 2015.

[11] X. Zhou, and L. Chen. An Incremental Local algorithm for better voltage control in distribution networks. Submitted to 55nd IEEE Annual Conference on Decision and Control (CDC), 2016.

[12] Standards Coordinating Committee 21 of Institute of Electrical and Electronics Engineers, Inc., IEEE Standard P1547.8 ${ }^{\mathrm{TM}} / \mathrm{D} 8$. Recommended practice for establishing methods and procedures that provide supplemental support for implementation strategies for expanded use of IEEE Standard 1547. IEEE ballot document, Aug 2014.

[13] Institute of Electrical and Electronics Engineers, Inc., IEEE Standard $1547 \mathrm{a}^{\mathrm{TM}}(2014)$. Standard for interconnecting distributed resources with electric power systems Amendment 1, May 2014.

[14] M. Farivar and S. H. Low. Branch flow model: Relaxations and convexification (parts I, II). Power Systems, IEEE Transactions on, 28(3):25542572, 2013.

[15] J. Shihadeh, S. You, and L. Chen. Signal-anticipating in local voltage control in distribution systems. Smart Grid Communications (SmartGridComm), 2014 IEEE International Conference on, 2014.

[16] L. Gan, and S. H. Low. An online gradient algorithm for optimal power flow on radial networks. IEEE Journal on Selected Areas in Communications, 34(3), March 2016.

[17] D. Emiliano, and A. Simonetto. Optimal power flow pursuit. arXiv preprint arXiv: 1601.07263 (2016).

[18] R. D. Zimmerman, C. E. Murillo-Snchez, and R. J. Thomas, MATPOWER: Steady-state operations, planning and analysis tools for power systems research and education. Power Systems, IEEE Transactions, vol. 26, no. 1, pp. 12-19, Feb. 2011.

[19] D. P. Bertsekas, and J. N. Tsitsiklis, Parallel and distributed computation: numerical methods. Vol. 23. Englewood Cliffs, NJ: Prentice hall, 1989. 